\title{
Cardiovascular Benefits of Testosterone Replacement Therapy in the Andropausal Male
}

\author{
Satya K. Vatti' ${ }^{1}$ Raef Madanieh11, Abed Madanieh'1, Constantine E. Kosmas², \\ Timothy J. Vittorio ${ }^{{ }^{*}}$ \\ ${ }^{1}$ St. Francis Hospital-The Heart Center ${ }^{\circledR}$, Division of Cardiology, Center for Advanced Cardiac Therapeutics, \\ Roslyn, NY, USA \\ ${ }^{2}$ Zena and Michael A Wiener Cardiovascular Institute, Icahn School of Medicine at Mount Sinai, New York, USA \\ Email: ${ }^{*}$ vittorio@hotmail.com
}

Received 9 June 2015; accepted 22 September 2015; published 25 September 2015

Copyright $@ 2015$ by authors and Scientific Research Publishing Inc.

This work is licensed under the Creative Commons Attribution International License (CC BY). http://creativecommons.org/licenses/by/4.0/

(c) (i) Open Access

\begin{abstract}
Androgen deficiency has been receiving a lot of attention as an evolving health concern in the industrialized world. With population aging, hypogonadism has become a challenging medical problem confronting practicing physicians. The importance of this increasingly prevalent disease arises from its potentially debilitating and serious consequences, such as atherosclerosis, diabetes mellitus, metabolic disorders, muscular weakness, psychiatric illness, sexual dysfunction and vascular inflammation. When left untreated, testosterone deficiency is associated with increased cardiovascular mortality.
\end{abstract}

\section{Keywords}

Cardiovascular, Hormones, Hypogonadism, Testosterone, Therapy

\section{Introduction}

Testosterone (T) is the primary androgen in the human body and it is assayed as Total Testosterone (TT). TT is composed of free testosterone (FT), sex hormone binding globulin-bound testosterone (SHBG-Bound T), and albumin-bound testosterone (A-bound T). Secondary androgens found in-vivo include but are not limited to androstenediol and androstenedione. Primary hypogonadism is a defect of the gonads, which results in low production of $\mathrm{T}$. Serum levels of $\mathrm{T}$ can also be affected in secondary hypogonadism, which results from decreased

"Corresponding author.

How to cite this paper: Vatti, S.K., Madanieh, R., Madanieh, A., Kosmas, C.E. and Vittorio, T.J. (2015) Cardiovascular Benefits of Testosterone Replacement Therapy in the Andropausal Male. Health, 7, 1206-1214.

http://dx.doi.org/10.4236/health.2015.79135 
levels of follicle-stimulating hormone (FSH), luteinizing hormone (LH) or gonadotropin-releasing hormone (GnRH). T production naturally declines in men every year after the age of 35 [1]. This steady decline in $\mathrm{T}$ can affect sex-specific characteristics and subsequently influence quality of life, since androgenic hormones are primarily responsible for stimulating, controlling and maintaining the male's sexual drive and spermatogenesis. In our review, we briefly discuss testosterone physiology, hypogonadism and pharmacokinetics of different available testosterone formulations, then we examine the evidence supporting the cardiovascular (CV) benefits that would ensue with each type of treatment by specifically discussing effects on cardiovascular hemodynamics, metabolic parameters and systemic inflammatory markers.

\section{Testosterone Physiology and Pathology}

$\mathrm{T}$ is the end-product of a series of hormonal interactions in the body. GnRH is secreted by the arcuate nucleus of the hypothalamus, which controls the secretion of FSH and LH by the adenohypophysis. FSH stimulates spermatogenesis by the seminiferous tubules, whereas LH regulates production and secretion of $\mathrm{T}$ by the Leydig cells of the testes. An elevated serum $\mathrm{T}$ concentration promotes feedback inhibition of further $\mathrm{T}$ production by decreasing levels of GnRH, FSH and LH. This delicate homeostasis is carefully maintained by the hypothalamic-hypophyseal-gonadal axis (HHG Axis). Additionally, T production is adversely affected by prolactin (PL) secreted by the lactotroph cells of the adenohypophysis. However, dopamine secreted by the hypothalamic periventricular zone inhibits PL production through the tuberoinfundibular pathway by binding to $\mathrm{D}_{2}$ receptors on the lactotroph membrane [2].

When there is a loss of $\mathrm{T}$ production at the gonadal level, the type of hypogonadism is classified as primary. Primary hypogonadism is diagnosed by increased serum levels of FSH and LH. This condition may occur naturally with aging. Nevertheless, it can also be caused by chemotherapy, cryptorchidism, Klinefelter's Syndrome and orchitis [3]. On the other hand, a reduction in T production due to any disease that can affect the hypothalamus or hypophysis is classified as secondary hypogonadism. Secondary hypogonadism often occurs in the presence of low or normal FSH and LH levels, and is commonly seen in chronic renal failure (CRF), diabetes mellitus (DM) and less commonly inhemochromatosis, hypophyseal mass lesions, obesity and prolactinomas [3].

An increased concentration of estradiol (E2), a form of estrogen, has a negative feedback effect on GnRH, FSH and LH. Estrogens such as E2 can also indirectly stimulate PL release through inhibition of dopamine synthesis. Adipocytes use the CYP19A1 aromatase enzyme to convert T to E2 [4]. Elevated levels of E2 can inhibit $\mathrm{T}$ production, thereby leading to a low $\mathrm{T}$ level. A well-known cause of decreased $\mathrm{T}$ levels and increased E2 levels in men is obesity, and in particular android obesity ("belly fat"). In fact, an elevated body mass index (BMI) has been directly correlated with proportionately decreased T levels [5]. Interestingly, some industrial chemicals can also increase E2 levels. Exposure to estrogenic chemicals, such as bisphanol A (BPA) found in plastics and the linings of canned food can lead to depressed $\mathrm{T}$ level and inhibition of spermatogenesis [6].

Most of $\mathrm{T}$ is bound to either SHBG or albumin in the serum, and the respective levels of each can also influence T levels. It is estimated that only $0.5 \%$ - $3 \%$ of TT is unbound and biochemically free. The concentration of SHBG in serum increases as males age with subsequent reduction of the levels of circulating FT. Circulating SHBG can be increased by elevated E2, liver disease and thyroxine (thyroid hormone), while it can be decreased by administration of androgens and glucocorticoids, and in chronic diseases such as chronic renal failure and DM. Since SHBG is an important determinant of T activity, there are other factors that must be assessed in order to determine the cause of low T levels. Craniopharyngiomas, hemochromatosis, meningiomas, sarcoidosis and tuberculosis can all inhibit GnRH secretion and cause a tertiary decrease of T levels if the hypothalamus or secondary decrease if the hypophysis is affected [7]. Drugs that can cause potentially reversible hypogonadism include metoclopramide and opioids, by inducing hyperprolactinemia [2]. Reversible hypogonadism can also be caused by exogenous steroids and GnRH agonists and antagonists, which are used in androgen deprivation therapy [8].

\section{Diagnosis of Male Hypogonadism}

Symptoms that should raise a clinical suspicion of low $\mathrm{T}$ levels include decreased libido, erectile dysfunction, fatigue and mood changes. Signs of T deficiency include decreased muscle mass (sarcopenia) and loss of axillary and pubic hair. Anemia, higher than normal percentages of body fat, impaired cognition, mood lability and osteopenia/osteoporosis are other important signs found in hypogonadal individuals [9]. 
Quantification of T concentration can be achieved by using three main methods: sampling blood, urine or saliva. Electrochemiluminescent Immunoassay (ECLIA) and Liquid Chromatography/Mass Spectrometry (LC/MS) are the most prevalent methods for assaying TT levels. Although there is concordance on the biochemical method of assay, there is a lack of accord on the normal parameters of plasma TT levels. The Endocrine Society suggests a cut-off of $300 \mathrm{ng} / \mathrm{dL}$ as a benchmark for treatment, while the American Association of Clinical Endocrinologists proposes a TT level of $200 \mathrm{ng} / \mathrm{dL}$ as a defining point for therapy. More recently, the European Association of Urology has deemed patients with a TT level of $230 \mathrm{ng} / \mathrm{dL}$ or less and typical symptoms to benefit the most from exogenous $\mathrm{T}$ supplementation, while, at the same time, urging physicians to start patients on a trial of testosterone replacement therapy (TRT) if TT levels are between 230 - 350 ng/dL and they present with symptoms [10]. Other tests to confirm the diagnosis of hypogonadism are serum FSH, LH, PL and SHBG levels. Further work-up may include brain magnetic resonance imaging (MRI) of the hypophysis and hypothalamus to exclude endocrine tumors, a karyotype analysis to exclude developmental disorders such as Klinefelter's syndrome and serum iron levels to detect possible hemochromatosis.

\section{Types of Testosterone Therapies}

Pure $\mathrm{T}$ is rarely used as a form of therapy and esters are usually added to avoid immediate absorption. Esters also help to release $\mathrm{T}$ in a controlled manner. For example, long-acting intramuscular (IM) testosterone enanthate (TE) or testosterone cypionate (TC) must be dosed every one to four weeks. While IM T is popular among patients, other modes of TRT include gel and patch forms of T. The gel and patch must be applied once and twice daily, respectively. Testosterone Undecanoate (TU) oral therapy is being prescribed in other countries but is not approved for patient use in the United States [3] [11].

\section{Pharmacokinetics of Testosterone Treatment}

The pharmacokinetics of several T modalities have been studied extensively in recent years. Studies have examined the effects of the Transdermal System for Testosterone (TDS-T), TE, TU and Sublingual Testosterone (SLT) on various parameters such as serum T levels, area under the curve (AUC), maximum concentration of $\mathrm{T}$ (Cmax), average concentration of $\mathrm{T}$ (Cavg) and time to maximum $\mathrm{T}$ concentration (Tmax).

The pharmacokinetics of TDS-T (50 mg dose) were investigated using a double-blind cross-over study that utilized Androgel 1\% (50 mg) and a TDS-placebo. Approximately 12 hours after administration, pharmacokinetic parameters such as AUC, which measures the bioavailability and absorption, Cmax (a higher maximum concentration of $\mathrm{T}$ is beneficial), and the Tmax (a higher time to maximum concentration is desired) were also measured [12]. The mean values of AUC, Cmax and Tmax for TDS-T were all greater than those of either Androgel or the placebo during the 12-hour follow-up period.

A comparison of 2.5 and $5 \mathrm{mg}$ doses of SLT administered three times daily (tid), and $200 \mathrm{mg}$ of IM TE dosed every 20 days, was conducted for 60 days. Serum T levels were found to be increased immediately after administering SLT and the levels rose to a peak after 20 minutes [13]. Subsequently, the T levels started to decline until they reached baseline in 360 minutes. After $200 \mathrm{mg}$ of TE supplementation, serum T levels rose to a peak in 2 - 4 days and steadily declined through day 20. The peak T levels achieved with TE were noted to be lower than those observed with SLT. Peak T levels in the SLT cohort subsequently declined to baseline, while the decline from peak $\mathrm{T}$ levels for the TE cohort did not reach baseline levels. Values of net AUC for the TE study group were significantly higher than the values for the SLT groups, indicating an increased serum Cavg T levels for subjects receiving TE.

\section{Contraindications and Side-Effects of Testosterone Replacement Therapy}

Before initiating TRT, a detailed medical history should be taken and a thorough physical examination should be performed. The prostate gland must be evaluated by a digital rectal exam (DRE), and pre-treatment prostatespecific antigen (PSA) levels should be measured. A lipid panel and liver function tests (LFTs) must also be obtained [3]. Patients should have morning T levels measured within 2 - 3 weeks of starting therapy with the patch or the gel and within 8 weeks if they are on the injectable form of TRT. Follow-up blood results should be obtained at 3 and 6 months [3].

There exists controversy over TRT, as it has been linked to growth of androgen-sensitive carcinomas [7]. 
However, it has been shown that low endogenous T levels have been clinically linked to aggressive metastatic prostatic carcinomas. Other relative contraindications include history of treatment for severe benign prostatic hypertrophy (BPH) and urinary outflow tract obstruction symptoms. Patients who have elevated PSA levels or are undergoing treatment for sleep-disordered breathing, such as obstructive sleep apnea syndrome, are cautioned against initiating therapy [14]. Erythrocytosis or secondary polycythemia is a known side-effect of TRT. It is advisable to stop therapy if the hematocrit rises above 54\% - 55\% [7]. Acne, fluid retention and gynecomastia are other potential side-effects that have been observed in patients at the onset of treatment [7].

Patients may be at risk for the development of pulmonary and deep venous thrombi while on TRT due to the presence of predisposing factors [15]. To offset this risk, it is recommended that these individuals adhere to an aspirin regimen for the duration of therapy.

Although some studies have postulated that TRT increases the risk of MI, recent research has shown that this might not necessarily be the case. Baillargeon, et al provided evidence that patients with the highest pre-study risk of MI had a decline in risk with TRT [16]. However, TRT did not have the same effect in patients with a low risk of MI. Subjects were classified into quartiles based on their risk. The Hazard Ratios (HR) for the highest risk quartile of the study population was 0.69 (95\% CI: 0.53 - 0.92), while the first, second and third quartiles had HRs of 1.20 (95\% CI: 0.88 - 1.67), 0.94 (95\% CI: 0.69 - 1.30), and 0.78 (95\% CI: 0.59 - 1.01), respectively [16].

While our review's main scope is to review the cardiovascular protective benefits of TRT in hypogonadal patients at a low risk for cardiovascular (CV) events, it is imperative to discuss two of the largest trials addressing the risk of CV events associated with TRT in patients with established coronary artery disease (CAD) and those at higher risk for MI.

A study by Finkle et al included a cohort of 55,593 patients prescribed TRT [17]. The data was extracted from a healthcare database and only evaluated the risk of nonfatal MI as the endpoint. The diagnosis was retrospectively extracted via ICD codes rather than structured evaluation. Moreover, the indications for which TRT was prescribed were not evaluated and serologic assessment was not done (FT and TT levels). Nonetheless, the study concluded the risk of nonfatal MI was remarkably increased in younger adults with pre-existing heart disease.

Vigen et al addressed the shortcomings of the previous study by evaluating all cause mortality, MI and cerebrovascular accidents (CVAs) as endpoints specifically in hypogonadal veteran men with a TT level $<300$ $\mathrm{ng} / \mathrm{dL}$ and who had angiographic evidence of CAD $(n=8709)$ [18]. However, this retrospective study had major shortcomings. The population of the study had a high burden of comorbidities putting this cohort at a very high risk for CAD (20\% had prior MI, 50\% with DM, and more than $80 \%$ with prior CAD). In the CAD patients receiving TRT only, 23 had a MI and 33 had an ischemic stroke. At 3 years follow-up, event rates were $19.9 \%$ in the non-TRT group $(n=7486)$ versus $25.7 \%$ in the TRT group $(n=1223)$. Furthermore, it is worth mentioning here that extended follow-up occurred in a relatively small number of 257 patients, making the generalizabilty of the study results limited.

\section{Cardiovascular Benefits of Testosterone Replacement Therapy}

In this section, we will discuss several studies that investigated the effects of various types of TRT such as Sustanon $100 \mathrm{mg}, 1 \%$ transdermal gel combined with recombinant human growth hormone (HGH), transdermal testosterone and NEBIDO (Active Ingredient: DHEA Sulfate $50 \mathrm{mg}$, TE $250 \mathrm{mg}$ and TU $160 \mathrm{mg}$ ) on cardiovascular (CV) parameters.

\subsection{Effects of Testosterone on Hemodynamic and Vascular Parameters}

A placebo-controlled trial assessed the effect of Sustanon $100 \mathrm{mg}$ on 10 patients with a mean age of 60.8 years. All patients carried a diagnosis of ischemic heart disease concomitantly with hypogonadism. After 4 weeks of treatment with either the placebo or Sustanon $100 \mathrm{mg}$ orally, the study examined the effects on selected variables. The time to $1 \mathrm{~mm}$ ST-segment depression was significantly increased $(\mathrm{p}=0.002)$ by an average of $74 \pm$ $54 \mathrm{sec}$ in patients being treated with Sustanon, as compared with placebo. A prolonged time to $1 \mathrm{~mm}$ ST-segment depression implies that the subject can engage in significant strenuous activity for a longer time without symptoms of angina or evidence of myocardial ischemia [19].

Mathur et al. utilized NEBIDO (TU) on 13 male patients with chronic angina to determine its effect on inducible ischemia threshold and on measures of atherosclerosis. Variables were measured at 14, 28 and 52 weeks. 
The time to ST-segment depression increased to $129.3 \pm 48 \mathrm{sec}$ after the 52 nd week of treatment versus $12 \pm 18$ sec with placebo $(\mathrm{p}=0.0001)$, which is indicative of increased CV fitness. Additionally, metabolic equivalents (METS), which is an indication of peak work load and physical strength, showed an increase of $1.4 \pm 0.72$ (p = 0.004) after 52 weeks of Nebido therapy [20].

Furthermore, the investigators demonstrated that the average carotid intima media thickness (CIMT) significantly decreased by $0.48 \pm 0.1 \mathrm{~mm}$ in 52 weeks $(\mathrm{p}=0.002)$. An increased CIMT is classically associated with an increased risk of vascular disease, including CAD and myocardial infarction (MI). Initiating an effective TRT regimen should reduce CIMT and thus reduce CV risk factors [20].

A clinical study conducted to determine T's effects on various arterial parameters recruited 18 males with a mean age of 62.5 years who presented with untreated hypogonadism. The hypothesis was that transdermal $\mathrm{T}$ gel had the potential to reduce the risk of developing CV disease. Transdermal $\mathrm{T}$ gel was administered over a 12-week period. Some variables associated with CAD risk, such as pulse wave velocity (PWV) and C1 (a measure of arterial compliance or elasticity), were measured at 48 hours, 6 weeks and 12 weeks of therapy. PWV is a factor of arterial elasticity and measures the pressure waves generated by the movement of blood. An inflexible artery causes an increase in the rate of deflection of the pressure wave, which results in an increase in the PWV. A higher PWV indicates the presence of arterial stiffness and is a predictor of future CAD and arteriosclerosis. After transdermal T gel was applied every other day, PWV significantly declined $(p=0.03)$ from a mean of 8.9 $\pm 31 \mathrm{~m} / \mathrm{s}$ to $8.24 \pm 0.3 \mathrm{~m} / \mathrm{s}$ and remained relatively constant for the remainder of the 12 weeks of the study. C1 increased $(\mathrm{p}=0.03)$ from a mean of $22.5 \pm 1.22 \mathrm{~mL} / \mathrm{mmHg} \times 10$ at baseline to $28.8 \pm 1.46 \mathrm{~mL} / \mathrm{mmHg} \times 10 \mathrm{after}$ 12 weeks of transdermal T gel treatment [21].

In another trial, DHEA Sulfate (50 mg) was used on 129 subjects with an average age of 70 years in a double-blind clinical study for a period of 12 months [22]. DHEA Sulfate is a potent androgen that seems to have significant CV benefits. To measure the effectiveness of DHEA Sulfate, arterial stiffness parameters such as PWV and Augmentation Index (AI) were measured. Increased blood flow during ventricular contraction causes an elevated PWV and an influx of excess blood. The excessive influx causes a significant rise in the central venous pressure (CVP). Since AI is the ratio of augmented CVP to pulse pressure, high CVP causes a significant increase in AI, which is an important predictor of CV morbidity and mortality [23]. AI is derived using a micromanometer that reduces irregularities on the surface of the right radial artery. The smooth surface is then used to calculate circumferential pressures that are combined to form a pressure wave form. After several wave forms are acquired, software is used to generate a cumulative wave form [24]. The contribution of the wave form reflection to the overall arterial pressure waveform is measured by AI. The PWV was reduced by an average of $3.5 \pm 1 \mathrm{~m} / \mathrm{s}$ after 12 months of therapy in comparison to placebo $(\mathrm{p}=0.001)$. When compared with the placebo, the AI declined by a mean of $6 \pm 2$ AI units with DHEA therapy for 12 months $(p=0.002)$ [22].

The association between serum $\mathrm{T}$ levels and atherosclerosis was further emphasized in a cross-sectional study that examined CIMT and endothelial function in 115 subjects with a mean age of 56.6 years [25]. The average CIMT was measured to be $0.107 \pm 0.01 \mathrm{~cm}$ in patients with a low concentration of T versus $0.083 \pm 0.01 \mathrm{~cm}$ in patients who had optimal levels (control) [25]. The measured difference in mean CIMT between both groups was significant with a p $<0.001$ [23]. Increased CIMT was prevalent in $80 \%$ and 39\% of low $\mathrm{T}$ and control groups, respectively [25].

Patients also underwent endothelial function assessment by brachial artery flow-mediated vasodilatation (FMD). An ultrasound transducer was used to obtain images of the brachial artery at baseline and at 60 sec after promulgation of reactive hyperemia by the use of a sphygmomanometer for a 5-minute time period. Patients with endothelial dysfunction had average TT levels of $13.52 \pm 4.74 \mathrm{nmol} / \mathrm{L}$, while patients who had no detectable dysfunction had a mean TT level of $18 \pm 4.74 \mathrm{nmol} / \mathrm{L}$. Incidence of endothelial dysfunction was $80.5 \%$ and $42.3 \%$ in the low $\mathrm{T}$ and control groups, respectively [25].

\subsection{Effects of Testosterone Therapy on Anthropometric and Metabolic Parameters}

Metabolic syndrome in males is defined according to the International Diabetes Federation Criteria (IDFC) which include: central obesity with waist circumference (WC $\geq 94 \mathrm{~cm} / 37$ in), serum Triglyceride (TG) level $>$ $150 \mathrm{mg} / \mathrm{dL}$ or treatment for hypertriglyceridemia, High Density Lipoprotein (HDL) $<40 \mathrm{mg} / \mathrm{dL}$ or treatment for reduced HDL, Systolic Blood Pressure $(\mathrm{SBP}) \geq 130 \mathrm{mmHg}$, diastolic BP (DBP) $>85 \mathrm{mmHg}$ or treatment for diagnosed hypertension (HTN) and the presence of type II DM or fasting glucose levels > $100 \mathrm{mg} / \mathrm{dL}$. Many 
previous studies have established a negative correlation between the severity of metabolic syndrome and low serum $\mathrm{T}$ levels. One of the important diagnostic criteria for metabolic syndrome is an increased WC, which is directly a result of increased adiposity. Adipocytes have been shown to secrete inflammatory markers such as tumor necrosis factor-alpha (TNF- $\alpha$ ) and interleukin-6 (IL-6), which may promote the development of insulin deficiency, atherogenic dyslipidemia and HTN. Thus, metabolic syndrome is a potent risk factor for cardiovascular disease [26].

For a better understanding of the effects of $\mathrm{T}$ on metabolic syndrome, 170 hypogonadal men with an average age of 51.6 years were enrolled in a double-blinded, placebo-controlled study for a 30-week duration. Anthropometric parameters, such as WC, hip circumference and waist-to-hip ratio, were examined along with biochemical assays of cholesterol, glucose, insulin and leptin levels. After 30 weeks of treatment with $1 \mathrm{gm} \mathrm{IM} \mathrm{TU}$, BMI decreased from a mean of $35.3 \mathrm{~kg} / \mathrm{m}^{2}$ (95\% CI: 34.2 - 36.6) at baseline to a mean of $34.1 \mathrm{~kg} / \mathrm{m}^{2}$ (95\% CI: 33.0 - 35.2). The $1.32 \mathrm{~kg} / \mathrm{m}^{2}$ change from baseline was significant $(\mathrm{p}<0.001)$. The weight $(\mathrm{kg})$ also declined from a mean of $115.1 \mathrm{~kg}$ at baseline (95\% CI: 110.6 - 119.6) to a mean of $110.8 \mathrm{~kg}$ (95\% CI: 106.3 - 115.3) over 30 weeks ( $<$ <.001). WC declined from a mean of $118 \mathrm{~cm}$ (95\% CI: $115.4-120.8)$ at baseline to $112.2 \mathrm{~cm}(95 \%$ CI: 109.7 - 114.7) after 30 weeks of treatment with TU (p < 0.001) [26].

TG levels declined by a mean of $0.32 \mathrm{mmol} / \mathrm{L}$ at 30 weeks of treatment versus baseline. Conversely, HDL levels were elevated after treatment by an average of $0.076 \mathrm{mmol} / \mathrm{L}$ versus baseline. Serum glucose levels also decreased from $6.2 \mathrm{mmol} / \mathrm{L}$ to $5.9 \mathrm{mmol} / \mathrm{L}$ after 30 weeks of treatment [26].

When investigators treated patients with a regimen of Sustanon $100 \mathrm{mg}$, there was a significant additional reduction in total cholesterol by a mean of $0.41 \pm 0.54 \mathrm{mmol} / \mathrm{L}$ in patients that received the therapy over a 4-week period. Total cholesterol reduction reduces the risk of developing atherosclerosis and coronary artery disease (CAD) [19].

When $1 \%$ transdermal $\mathrm{T}$ gel with rHGH was utilized, there was a significant reduction of TGs and a significant increase in HDL cholesterol. More specifically, the mean decline in fasting TG was $18.2 \pm 57.0 \mathrm{mg} / \mathrm{dL}(\mathrm{p}=$ $0.001)$ and the mean HDL increase was $3.5 \pm 6.7 \mathrm{mg} / \mathrm{dL}(\mathrm{p}<0.001)$. SBP rose significantly $(\mathrm{p}<0.001)$ by $12 \pm$ $14 \mathrm{mmHg}$. It should also be noted that the Quantitative Insulin Sensitivity Check Index (QUICKI), which correlates the fasting glucose and insulin levels, showed a statistically significant $(p=0.003)$ decline of $0.004 \pm 0.015$ [27].

In a study, in which subjects were administered transdermal $\mathrm{T}$ gel for 48 hours, Low Density Lipoprotein-C (LDL-C) decreased significantly from a mean of $2.76 \pm 0.69 \mathrm{mmol} / \mathrm{L}$ at baseline to $2.65 \pm 0.41 \mathrm{mmol} / \mathrm{L}$ at 12 weeks $(\mathrm{p}=0.0005)[21]$.

Furthermore, Serum TG levels declined by an average of $15 \pm 6 \mathrm{mg} / \mathrm{dL}$ with DHEA Sulfate therapy $(\mathrm{p}=0.03)$ when compared with the placebo [22].

Studies have shown that $\mathrm{T}$ supplementation has a beneficial effect on anthropometric parameters such as body composition and weight. These parameters are known to have an indirect effect on CV health. Several formulations of $\mathrm{T}$ have been proven to decrease body fat, increase muscle strength and increase weight loss.

After 16 weeks of treatment with 1\% transdermal T gel combined with recombinant HGH versus placebo, the changes in anthropometric variables were examined on 112 men, aged between the ages of 65 and 90 years. The BMI increased by a mean of $0.16 \mathrm{~kg} / \mathrm{m}^{2}(\mathrm{p}=0.01)$ after 16 weeks, presumably due to an increase in lean body mass (LBM) of $1.79 \pm 1.89 \mathrm{~kg}(\mathrm{p}<0.001)$ and a corresponding increase of $0.85 \pm 1.15 \mathrm{~kg}(\mathrm{p}<0.001)$ in appendicular LBM. Simultaneously, there was a decline of $1.33 \pm 1.74 \mathrm{~kg}(\mathrm{p}<0.001)$ in total fat and $0.88 \pm 1.21 \mathrm{~kg}$ in truncal fat [27].

Body mass declined by $0.93 \pm 3.6 \mathrm{~kg}(\mathrm{p}=0.02)$ and BMI decreased significantly by $0.29 \pm 1.3 \mathrm{~kg} / \mathrm{m}^{2}$ during a 52-week period of NEBIDO therapy $(\mathrm{p}=0.03)$ [20].

When $1 \mathrm{gm}$ of TU was administered to 255 subjects, aged between 33 and 69 years over a 5-year period, T levels rose from an average of $9.9 \mathrm{nmol} / \mathrm{L}$ pre-treatment to $18 \mathrm{nmol} / \mathrm{L}$ after one year of therapy [28]. $\mathrm{T}$ levels seemed to reach a steady state after 12 months of therapy and additional therapy did not have an effect on TT levels. Over a 5-year period, TU seemed to produce significant weight loss. The average weight decreased from $106.22 \pm 16.93$ to $90.07 \pm 9.51 \mathrm{~kg}$ with a mean loss of $15.35 \pm 0.43 \mathrm{~kg}$ during the 5-year period. The reduction in weight was statistically significant $(\mathrm{p}<0.0001)$ for each year of the 5 years of the study. The WC declined from $107.24 \pm 9.14 \mathrm{~cm}$ at baseline to $98.46 \pm 7.39 \mathrm{~cm}$ after 5 years of treatment. The BMI decreased from $33.9 \pm$ 5.51 to $29.13 \pm 3.09 \mathrm{~kg} / \mathrm{m}^{2}$ over the 5-year span [28]. The rate of decline was higher during the first 2 years of treatment for weight, WC and BMI as compared with the last 3 years of treatment. 
The beneficial impact of $\mathrm{T}$ supplementation on body composition is also illustrated by a study that used a gel-containing $50 \mathrm{mg}$ of $\mathrm{T}$ on 23 subjects aged 60 to 78 years, while an equal number of similarly aged study participants received a comparable quantity of placebo gel [29]. The BMI in $\mathrm{kg} / \mathrm{m}^{2}$ and total LBM (kg) were measured at baseline, 3 months and 6 months. The BMI and LBM both increased from baseline at 3 months when compared with the placebo group. Of particular importance, LBM increased from $64.3 \pm 7.6 \mathrm{~kg}$ at baseline to $66.0 \pm 8.4 \mathrm{~kg}$ on average at the end of 6 months. The WC was not significantly impacted after either 3 or 6 months of treatment [29].

Using a $6 \mathrm{mg} /$ day patch of transdermal $\mathrm{T}$ on 96 males over the age of 65, researchers at the University of Pennsylvania found that the mean serum T concentration increased from $367 \pm 79 \mathrm{ng} / \mathrm{dL}$ prior to the treatment to $625 \pm 249 \mathrm{ng} / \mathrm{dl}$ after 6 months of treatment [30]. Percentage of fat mass and LBM were measured separately and categorized by anatomical areas. While there was a decrease in average fat mass in the T-treated group over a 36-month period, it was only significant in the extremities [arms $(-0.7 \pm 0.1 \mathrm{~kg} ; \mathrm{p}<0.001)$ and legs $(-1.1 \pm$ $0.2 \mathrm{~kg} ; \mathrm{p}<0.001)$ ], but not in the trunk. An increased average LBM was observed in the trunk area, but not in the extremities. Overall, LBM saw an increase of $1.9 \pm 2.0 \mathrm{~kg}$ on average after 36 months of therapy [30].

Similarly, when hypogonadal geriatric males received IM TE injections, LBM increased by a mean of $4.2 \pm$ $0.6 \mathrm{~kg}$. The increase in LBM was significant in the legs but was insignificant in the arms. Body fat percentage was also significantly reduced compared with the placebo group after six months of treatment [31].

\subsection{Effects of Testosterone Therapy on Inflammatory Markers}

Inflammatory cytokines, such as TNF- $\alpha$ and IL-6, induce endothelial inflammation which promotes atheroma proliferation and atherosclerosis [5]. The inflammatory cytokines TNF- $\alpha$ and IL-6 declined when 50 mg of DHEA supplementation was used in a 129 subject clinical trial. After 12 months, there was reduced vascular endothelial damage. TNF- $\alpha$ levels had a mean decline of $0.56 \pm 0.23 \mathrm{pg} / \mathrm{mL}^{-1}(\mathrm{p}=0.02)$, while IL-6 levels had a mean change of $-0.80 \pm 0.20 \mathrm{pg} / \mathrm{mL}^{-1}(\mathrm{p}=0.0001)$ [22].

TU therapy also had an effect on the markers of inflammation such as TNF- $\alpha$, highly sensitive C-Reactive Protein (hs-CRP) and interleukin-1-beta (IL-1 $\beta$ ). TNF- $\alpha$ and hs-CRP levels decreased significantly, while IL-1 $\beta$ declined by a smaller margin. When 170 hypogonadal men with a mean age of 51.6 years were treated with 1 gm IM TU for 30 weeks, the mean TNF- $\alpha$ level declined from $3.5 \mathrm{ng} / \mathrm{L}$ (CI: 2.8 - 4.4) to $2.4 \mathrm{ng} / \mathrm{L}$ (CI: 1.8 - 3.0), $\mathrm{p}=0.03$. The mean hs-CRP level decreased ( $<$ 0.001) from $29 \mathrm{mg} / \mathrm{L}$ (CI: 23 - 35) to $19 \mathrm{mg} / \mathrm{L}$ (CI: 15 - 24). The average IL-1 $\beta$ levels declined from 1.7 ng/L (CI: 1.4 - 2.1) pre-treatment to 1.5 ng/L (CI: 1.2 - 1.8$)$ post-treatment $(\mathrm{p}=0.008)$. Furthermore, hs-CRP levels were also elevated in the low $\mathrm{T}$ group. The average hs-CRP levels were measured to be $2.74 \pm 1.37 \mathrm{mg} / \mathrm{L}$ in the low $\mathrm{T}$ group, while levels were substantially lower at $0.89 \pm 0.93 \mathrm{mg} / \mathrm{L}$ in the control group [26].

\section{Conclusion}

Clinical studies have demonstrated antiatherogenic benefits from TRT, as therapy reduced atherosclerosis and global CAD risk factors. Some of these trials are limited in sample size and duration of therapy, however. Despite the previously held belief, there is compelling evidence showing TRT might decrease the risk of CV disease by improving risk factors. Currently, the challenge remains to diagnose hypogonadism and to overcome the misconception held by physicians and patients that associate TRT with CV disease. Further research in the form of large scale randomized controlled trials is still warranted to assess the risk and benefits in groups at different cardiovascular risk levels before wider application of TRT.

\section{References}

[1] Basaria, S. (2013) Reproductive Aging in Men. Endocrinology and Metabolism Clinics of North America, 42, $255-270$. http://dx.doi.org/10.1016/j.ecl.2013.02.012

[2] La Torre, D. and Falorni, A. (2007) Pharmacological Causes of Hyperprolactinemia. Therapeutics and Clinical Risk Management, 3, 929-951.

[3] Dana, O. and Susanne, Q. (2006) Clinical Hypogonadism and Androgen Replacement Therapy: An Overview. Urologic Nursing, 226, 253-259.

[4] Ullah, M.I., Riche, D.M. and Koch, C.A. (2014) Transdermal Testosterone Replacement Therapy in Men. Drug Design, 
Development and Therapy, 8, 101-112.

[5] Huhtaniemi, I. (2014) Late-Onset Hypogonadism: Current Concepts and Controversies of Pathogenesis, Diagnosis and Treatment. Asian Journal of Andrology, 6, 192-202. http://dx.doi.org/10.4103/1008-682X.122336

[6] Jeng, H.A. (2014) Exposure to Endocrine Disrupting Chemicals and Male Reproductive Health. Frontiers in Public Health, 22, 1-4. http://dx.doi.org/10.3389/fpubh.2014.00055

[7] Surampudi, P., Swerdloff, R.S. and Wang, C. (2014) An Update on Male Hypogonadism Therapy. Expert Opinion Pharmacotherapy, 15, 1247-1264. http://dx.doi.org/10.1517/14656566.2014.913022

[8] Bhasin, S., Cunningham, G.R., Hayes, F.J., Matsumoto, A.M., et al. (2010) Testosterone Therapy in Men with Androgen Deficiency Syndromes: An Endocrine Society Clinical Practice Guideline. Journal of Clinical Endocrinology and Metabolism, 95, 2536-2559. http://dx.doi.org/10.1210/jc.2009-2354

[9] Grober, E.D. (2014) Testosterone Deficiency and Replacement: Myths and Realities. Canadian Urological Association Journal, 8, s145-s147. http://dx.doi.org/10.5489/cuaj.2309

[10] Miner, M., Barkin, J. and Rosenberg, M.T. (2014) Testosterone Deficiency: Myths Facts and Controversy. Canadian Journal of Urology, Suppl. 2, 39-54.

[11] Handelsman, D.J. and Zajac, J.D. (2004) Androgen Deficiency and Replacement Therapy in Men. Medical Journal of Australia, 180, 529-535.

[12] Chik, Z., Johnston, A., Tucker, A.T., Chew, S.L., et al. (2005) Pharmacokinetics of a New Testosterone Transdermal Delivery System, TDS-Testosterone in Healthy Males. British Journal of Clinical Pharmacology, 61, 275-279. http://dx.doi.org/10.1111/j.1365-2125.2005.02542.x

[13] Salehian, B., Wang, C., Alexander, G., Davidson, T., et al. (1995) Pharmacokinetics, Bioefficacy, and Safety of Sublingual Testosterone Cyclodextrin in Hypogonadal Men: Comparison to Testosterone Enanthate-A Clinical Research Center Study. Journal of Clinical Endocrinology and Metabolism, 80, 3567-3575.

[14] Hellstrom, W.J.G. (2004) Testosterone Replacement Therapy. Scientific World Journal, 4, 142-149. http://dx.doi.org/10.1100/tsw.2004.60

[15] Holmegard, H.N., Nordestgaard, B.G., Schnohr, P., Tybjaerg-Hansen, A. and Benn, M. (2014) Endogenous Sex Hormones and Risk of Venous Thromboembolism in Women and Men. Journal of Thrombosis and Haemostasis, 12, 297305. http://dx.doi.org/10.1111/jth.12484

[16] Baillargeon, J., Urban, R.J., Kuo, Y.F., Ottenbacher, K.J., et al. (2014) Risk of Myocardial Infarction in Older Men Receiving Testosterone Therapy. Annals of Pharmacotherapy, 48, 1138-1144. http://dx.doi.org/10.1177/1060028014539918

[17] Finkle, W.D., Greenland, S., Ridgeway, G.K., Adams, J.L., Frasco, M.A., et al. (2014) Increased Risk of Non-Fatal Myocardial Infarction Following Testosterone Therapy Prescription in Men. PLoS ONE, 9, e85805. http://dx.doi.org/10.1371/journal.pone.0085805

[18] Vigen, R., O’Donnell, C.I., Baron, A.E., Grunwald, G.K., Maddox, T.M., et al. (2013) Association of Testosterone Therapy with Mortality, Myocardial Infarction, and Stroke in Men With Low Testosterone Levels. Journal of the American Medical Association, 310, 1829-1836. http://dx.doi.org/10.1001/jama.2013.280386

[19] Malkin, C.J., Pugh, P.J., Morris, P.D., Kerry, K.E., Jones, R.D., Jones, T.H. and Channer, K.S. (2004) Testosterone Replacement in Hypogonadal Men with Angina Improves Ischemic Threshold and Quality of Life. Heart Journal, 90, 871-876. http://dx.doi.org/10.1136/hrt.2003.021121

[20] Mathur, A., Malkin, C., Saeed, B., Muthusamy, R., Jones, T.H. and Channer, K. (2009) Long-Term Benefits of Testosterone Replacement Therapy on Angina Threshold and Atheroma in Men. European Journal of Endocrinology, 161, 443-449. http://dx.doi.org/10.1530/EJE-09-0092

[21] Yaron, M., Greenman, Y., Rosenfeld, J., Izkhakov, E., Limor, R., et al. (2009) Effect of Testosterone Replacement Therapy on Arterial Stiffness in Older Hypogonadal Men. European Journal of Endocrinology, 160, 839-846. http://dx.doi.org/10.1530/EJE-09-0052

[22] Edward, W., Dennis, V., Ali, E., Luigi, F. and John, H. (2012) Dehydroepiandrosterone Replacement Therapy in Older Adults Improves Indices of Arterial Stiffness. Aging Cell Journal, 11, 876-884. http://dx.doi.org/10.1111/j.1474-9726.2012.00852.x

[23] Nichols, W.W. and Singh, B.M. (2002) Augmentation Index as a Measure of Peripheral Vascular Disease State. Current Opinion in Cardiology, 17, 543-551. http://dx.doi.org/10.1097/00001573-200209000-00016

[24] Poongothai, S., Pradeepa, R., Indulekha, K., Surendar, J., et al. (2015) Association of Depression with Common Carotid Artery Intima Media Thickness and Augmentation Index in a Large Urban South Indian Population-The Chennai Urban Rural Epidemiology Study (CURES-138). Indian Journal of Endocrinology and Metabolism, 19, 136-142. 
http://dx.doi.org/10.4103/2230-8210.146869

[25] Farias, J.M., Tinetti, M., Khoury, M. and Umpierrez, G.E. (2014) Low Testosterone Concentration and Atherosclerotic Disease Markers in Male Patients with Type 2 Diabetes. Journal of Clinical Endocrinology and Metabolism, 99, 46984703. http://dx.doi.org/10.1210/jc.2014-2585

[26] Kalinchenko, S.Y., Tishova, Y.A., Mskhalaya, G.J., Gooren, L.J.G., et al. (2010) Effects of Testosterone Supplementation on Markers of the Metabolic Syndrome and Inflammation in Hypogonadal Men with the Metabolic Syndrome: The Double-Blinded Placebo-Controlled Moscow Study. Clinical Endocrinology, 73, 602-612. http://dx.doi.org/10.1111/j.1365-2265.2010.03845.x

[27] He, J., Bhasin, S., Binder, E.F., Yarasheski, K.E., et al. (2013) Cardio Metabolic Risks during Anabolic Hormone Supplementation in Older Men. Obesity Journal, 21, 968-975. http://dx.doi.org/10.1002/oby.20081

[28] Saad, F., Haider, A., Doros, G. and Traish, A. (2013) Long Term Treatment of Hypogonadal Men with Testosterone Produces Substantial and Sustained Weight Loss. Obesity, 21, 1975-1981. http://dx.doi.org/10.1002/oby.20407

[29] Frederiksen, L., Hojlund, K., Hougaard, D.M., Brixen, K. and Andersen, M. (2012) Testosterone Therapy Increased Muscle Mass and Lipid Oxidation in Aging Men. AGE, 34, 145-156. http://dx.doi.org/10.1007/s11357-011-9213-9

[30] Snyder, P.J., Peachey, H., Hannoush, P., Berlin, J.A., Loh, L., et al. (1999) Effect of Testosterone Treatment on Body Composition and Muscle Strength in Men over 65 Years of Age. Journal of Clinical Endocrinology and Metabolism, 84, 2647-2653.

[31] Ferrando, A.A., Sheffield-Moore, M., Yeckel, C.W., Gilkison, C., Jiang, J., et al. (2002) Testosterone Administration to Older Men Improves Muscle Function: Molecular and Physiological Mechanisms. American Journal of Physiology, Endocrinology and Metabolism, 282, E601-E607. http://dx.doi.org/10.1152/ajpendo.00362.2001 